\title{
A metallicity-flattening relation for dwarf elliptical galaxies
}

\author{
F. D. Barazza and B. Binggeli
}

Astronomisches Institut, Universität Basel, Venusstrasse 7, 4102 Binningen, Switzerland

Received 2 September 2002 / Accepted 5 September 2002

\begin{abstract}
It is shown that bright cluster dwarf ellipticals follow a relation between metallicity and apparent flattening. Rounder dwarfs tend to be more metal-rich. The evidence is based on colour as well as spectroscopic line-strength data from the literature. The large scatter of dEs around the mean metallicity-luminosity relation, usually ascribed to large observational errors, turns out to be an ellipticity effect. In the magnitude range $-14 \gtrsim M_{B} \gtrsim-18$ the metallicity of dEs depends more strongly on ellipticity than luminosity. A possible explanation is that galaxies with masses around $10^{9} M_{\odot}$ suffered a partial blowout of metal-enriched gas along their minor axis, rendering ellipticity a critical parameter for metallicity (De Young \& Heckman 1994).
\end{abstract}

Key words. galaxies: general - galaxies: fundamental parameters - galaxies: elliptical - galaxies: dwarf galaxies: evolution

\section{Introduction}

Elliptical galaxies follow a well-known luminosity-metallicity relation, in the sense that more luminous ellipticals are observed to be globally redder, or have larger metallic absorption line strengths in their spectra than less luminous systems (Faber 1973; Visvanathan \& Sandage 1977). Obviously this is a mass-metallicity relation, and its canonical interpretation is that less massive galaxies had more significant outflows of metal-enriched gas at an early evolutionary stage (Faber 1973; Mould 1984). The relation seems to hold for the entire luminosity range of spheroidal galaxies, down to the faintest dwarfs (Caldwell 1983; Brodie \& Huchra 1991; Caldwell et al. 1992). However, there is also considerable scatter in the relation, and there have been attempts to find a second parameter which governs metallicity (mass being the primary parameter). A good candidate is the internal velocity dispersion (Terlevich et al. 1984; Efstathiou \& Fall 1984), which even more strongly correlates with metallicity than mass (Bender et al. 1993).

The largest scatter in the luminosity-metallicity relation is observed in the magnitude range of bright cluster dwarf ellipticals $\left(-14 \gtrsim M_{B} \gtrsim-18\right)$. While this scatter could plausibly be due to measurement errors, Rakos et al. (2001) find a weak correlation with age for a sample of Fornax cluster dwarfs observed in Stroemgren narrow-band colours.

In this letter we show that the scatter in metallicity for cluster dEs is largely explained by apparent ellipticity (flattening). At a given luminosity, rounder dwarf ellipticals are more metal-rich. There have already been hints that ellipticity might act as a second parameter in normal elliptical galaxies (Terlevich et al. 1984). However, for dEs the effect is so

Send offprint requests to: F. D. Barazza,

e-mail: barazza@astro.unibas.ch strong that ellipticity appears to be the primary parameter. Such a metallicity-flattening relation for $\mathrm{dEs}$ is not implausible. The outflow of metal-enriched gas in stellar systems of intermediate mass $\left(M \approx 10^{9} M_{\odot}\right)$ is preferentially occurring along the minor axis, rendering ellipticity a critical parameter for the metallicity of present day dwarfs (De Young \& Heckman 1994). Rounder dEs seem to have suffered less significant outflow.

\section{Colour versus flattening}

We first show the evidence from multi-colour photometry, which is how we stumbled over the flattening effect. In Fig. 1 we have plotted integrated colour versus apparent ellipticity for different samples of spheroidal galaxies. The filled circles are $U-B$ values for 15 Virgo dEs from our own photometry (Barazza et al., in prep.). There is a very clear trend appearing, in the sense that rounder dEs are redder than more flattened ones. Such a correlation is rather unexpected - see for comparison a sample of normal ellipticals from Peletier et al. (1990, triangles). However, our finding is corroborated by the $U-B$ data for Virgo and Fornax dEs (crossed circles) from Caldwell (1983) and Caldwell \& Bothun (1987), and the Stroemgren $v z-y z$ data for Fornax dEs (open circles) from Rakos et al. (2001). In contrast, local dwarfs with colour data available (from Mateo 1998, asterisks) show no clear relation. All ellipticities used here are taken either from the references given for colour/metallicity data, or from LEDA.

Is the trend for cluster dEs real? Observational bias can be excluded: the samples were not selected by colour or ellipticity. Nor is it conceivable that the photometric errors in colour somehow depend on the ellipticity of the whole galaxy. Another suspicion is that ellipticity is not an independent parameter: if ellipticity were correlated with absolute magnitude, 


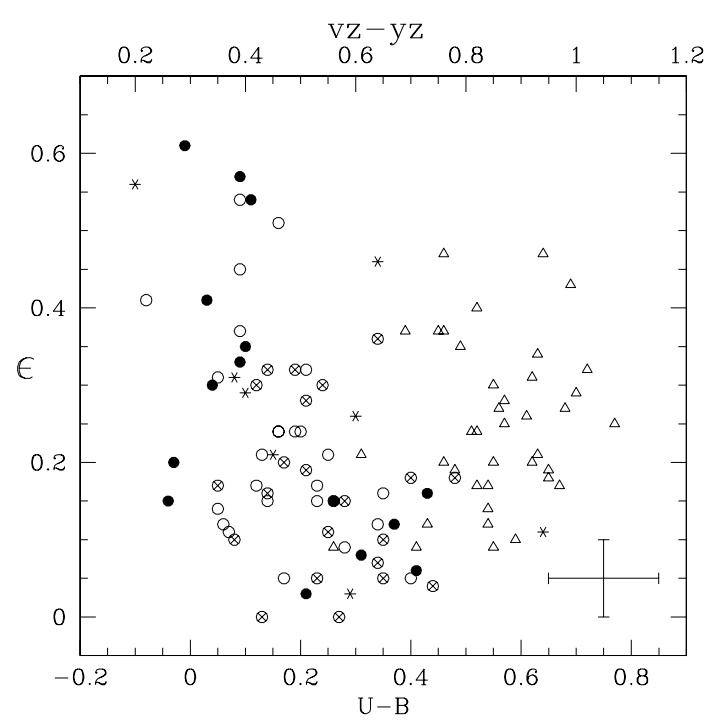

Fig. 1. Colour versus apparent ellipticity for cluster dEs (circles), local $\mathrm{dEs} / \mathrm{dSphs}$ (asterisks), and normal ellipticals (triangles). Filled circles are Virgo dEs from our own photometry, crossed circles are Virgo and Fornax dEs from Caldwell et al., open circles are Fornax dEs from Rakos et al. (complete references given in the text). For the Rakos et al. data the colour plotted is $v z-y z$ (Stroemgren system), shifted by an arbitrary amount along the abscissa (scale on top). For all other samples the conventional $U-B$ colour is plotted (bottom scale). Typical error bars are shown in the lower right.

we simply might have recovered the well-known luminositycolour relation (e.g. Ferguson 1994). However, the ellipticity of dEs is not significantly related to luminosity, nor to surface brightness (Binggeli \& Popescu 1995).

We take it for granted that colour is essentially indicating metallicity, as spheroidal galaxies are believed to be dust-free and old. Of course, metallicity can also be measured more directly. We now show that the colour-ellipticity relation is indeed a metallicity-ellipticity relation.

\section{The luminosity-metallicity relation}

Figure 2 shows the "universal" luminosity-metallicity relation for essentially the same galaxies as in Fig. 1. Normal Es (small triangles) are represented by data from a more recent study (Kobayashi \& Arimoto 1999). [Fe/H] values for local dwarfs (asterisks) are again from Mateo (1998). For bright cluster dEs $\left(-14 \gtrsim M_{B} \gtrsim-18\right)$ we have three sources for $\left.[\mathrm{Fe} / \mathrm{H}]: 1\right)$ Brodie \& Huchra (1991) give spectroscopically determined metallicities for nearly 40 Virgo and Fornax cluster dEs (essentially the Caldwell et al. sample) based on several different line indices. However, the estimated errors in $[\mathrm{Fe} / \mathrm{H}]$ are considerable. Data with $1 \sigma$ errors less than 0.6 dex are plotted as filled circles, those with larger errors (but smaller than 1 dex) as filled squares. 2) Held \& Mould (1994) provide spectroscopically measured $[\mathrm{Fe} / \mathrm{H}]$ values, with much smaller estimated errors, for 8 nucleated Fornax dEs (crossed circles). 3) Finally, there are the Rakos et al. (2001) $v z-y z$ Stroemgren colours for Fornax dEs, appropriately transformed into $[\mathrm{Fe} / \mathrm{H}]$ values (open circles), with a small mean error of 0.2 dex.

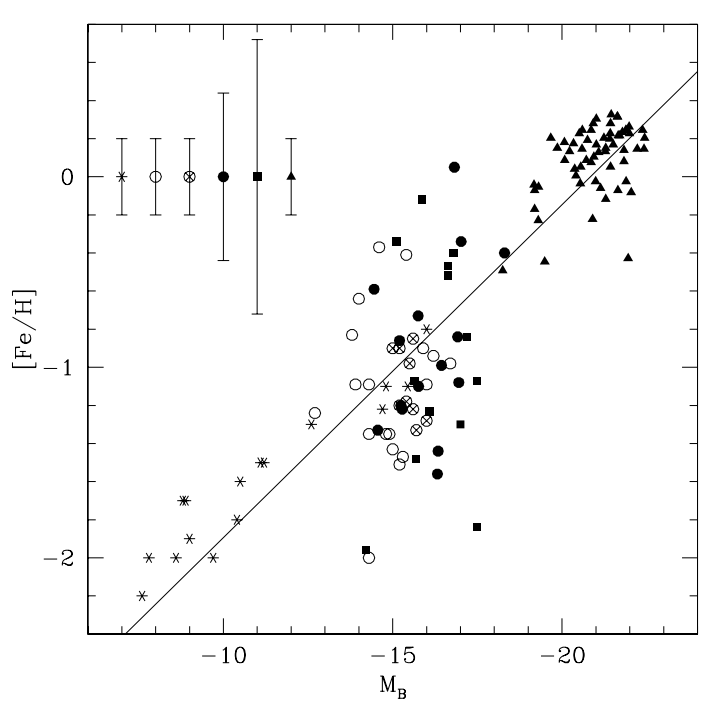

Fig. 2. $[\mathrm{Fe} / \mathrm{H}]$ versus absolute blue magnitude for cluster dEs (circles and squares), local dEs/dSphs (asterisks), and normal Es (small triangles). Open circles are estimated $[\mathrm{Fe} / \mathrm{H}]$ values for Fornax dEs based on Stroemgren colours (Rakos et al.), crossed circles are spectroscopically measured metallicities for Fornax dEs (Held \& Mould). Filled circles and squares are $[\mathrm{Fe} / \mathrm{H}]$ values for Virgo and Fornax dEs, spectroscopically determined as well (Brodie \& Huchra), but with considerable errors. Mean errors for the different samples are shown as error bars in the upper left corner. Complete references are given in the text. The line is a fit to all data (equation given in text).

Naively fitting a line through all data plotted in Fig. 2, we get an expression for the mean (universal) luminositymetallicity relation: $[\mathrm{Fe} / \mathrm{H}]=-0.1747 M_{B}-3.6405$, which will be used for a residual analysis below. The local dwarfs follow the mean relation surprisingly well. In contrast, the cluster dEs in the intermediate range between normal Es and extreme dwarfs, while falling in place with the universal relation in the mean, show enormous scatter in their individual metallicities. The natural suspicion is that this is simply due to large errors in $[\mathrm{Fe} / \mathrm{H}]$. However, we now show that this scatter is systematically related to ellipticity, i.e. it must, at least partially, be real.

\section{Flattening as second parameter}

The residual metallicities with respect to the line shown in Fig. 2 are plotted versus ellipticity in Fig. 3. There is clearly no correlation for normal Es and local dwarfs (upper panel), whereas a relation is evident for cluster dEs (lower panel): dEs with positive residuals are throughout round, while highly flattened dEs have always negative residuals. At a given luminosity, rounder dEs tend to be more metal-rich.

Several points have to be noted. First, the trend is followed by all $\mathrm{dE}$ samples used, even those where large errors were claimed (the Brodie \& Huchra data). Again it is not conceivable why these errors, were they real, should correlate with ellipticity (we believe the errors are simply overestimated). Second, we have tested that the metallicity residuals do not correlate with other parameters, such as effective radius, i.e. there is no 


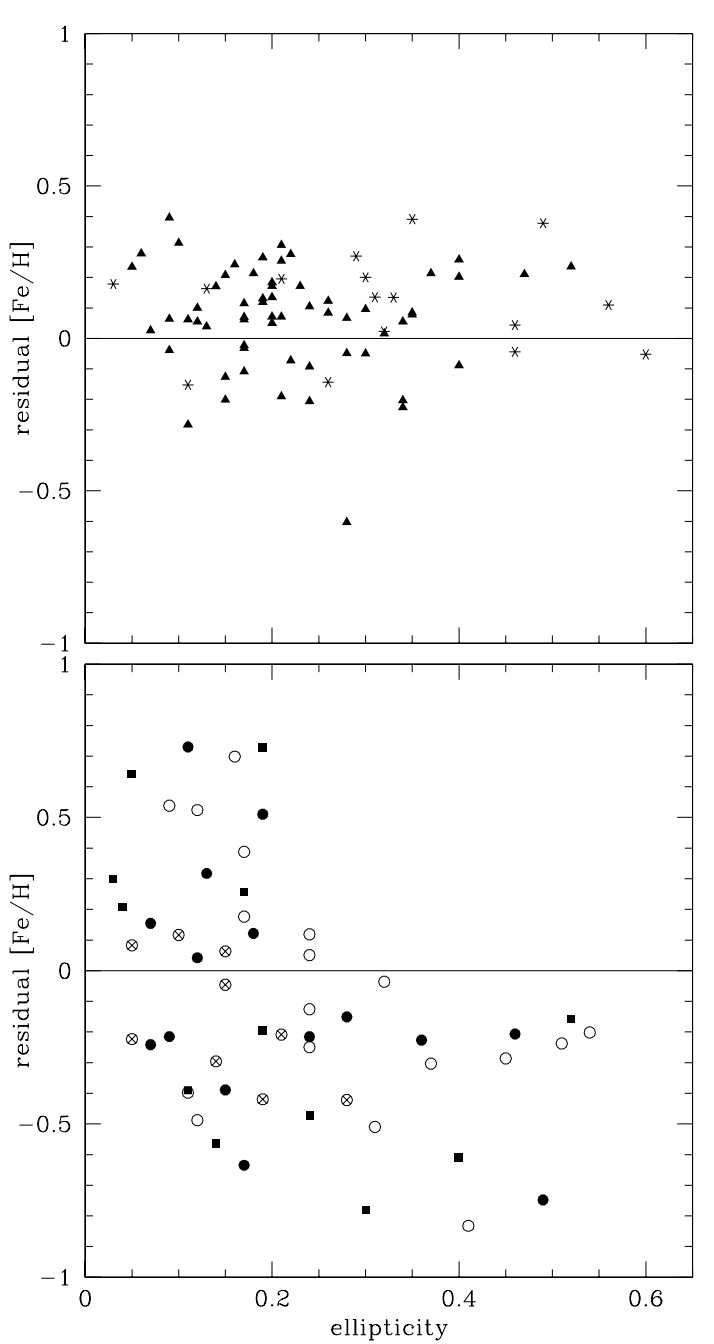

Fig. 3. Residual $[\mathrm{Fe} / \mathrm{H}]$ with respect to the linear fit in Fig. 2 versus apparent ellipticity for normal ellipticals and local dwarfs (upper panel), and for cluster dEs (lower panel). Symbols as in Fig. 2.

other "second" parameter than ellipticity. Third, the true physical relation behind this effect will involve intrinsic ellipticity. Due to random projection this relation should even be stronger than what we see with apparent ellipticity. The distribution of points in Fig. 3 (lower panel) is in accord with what we expect from projection effects: apparently round galaxies with positive residuals will also be intrinsically round, while some of the apparently round galaxies with negative residuals will intrinsically be more flattened (i.e. would be shifted to the right).

\section{The metallicity-flattening relation}

In Fig. 4 we have plotted metallicity directly versus ellipticity, to get an equivalent to the colour-ellipticity diagram of Fig. 1. As expected, no correlation is seen for giant Es, while dwarf objects evidently follow the suspected relation: rounder galaxies tend to be more metal-rich than flatter ones. Owing to some outliers, the trend is less evident for the local dwarfs (no correlation at all was seen in the residual plot). Conceivably, local (field) dwarfs are systematically different from cluster dwarfs (see Sect. 6).

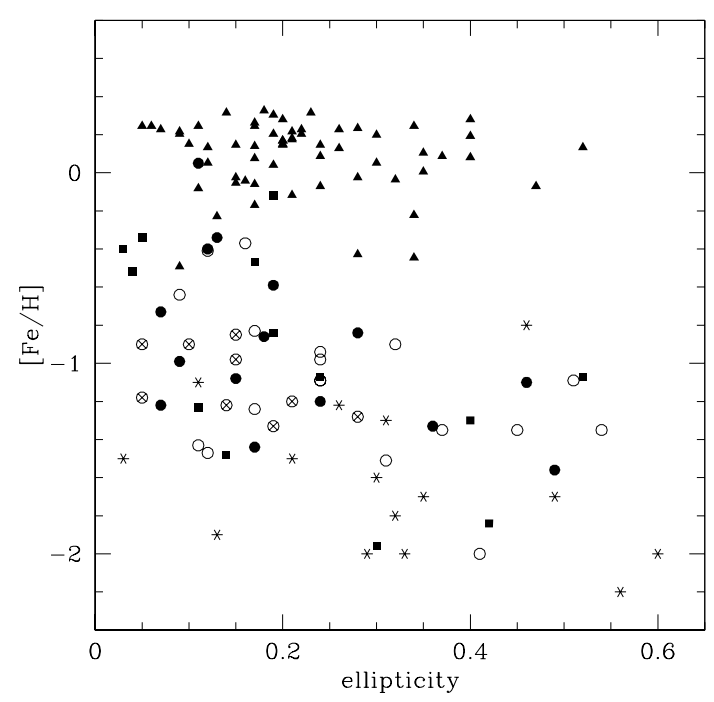

Fig. 4. $[\mathrm{Fe} / \mathrm{H}]$ versus apparent ellipticity. Symbols as in Fig. 2.

In general, the flattening-metallicity relation is not as striking as the residual plots in Fig. 3. This is because luminosity now acts as a hidden parameter, adding scatter. In Fig. 5 we have plotted metallicity versus logarithmic ellipticity for only cluster dwarfs, which provides an almost linear relation. A linear fit yields $[\mathrm{Fe} / \mathrm{H}]=-0.72 \log (\epsilon)-1.56$, or in terms of abundance: $Z / Z_{\odot} \propto \epsilon^{-0.72}$. Including the absolute magnitude and fitting a plane to the cluster dwarfs in the parameter space defined by $[\mathrm{Fe} / \mathrm{H}], \epsilon$ and $M_{B}$, we get $Z / Z_{\odot} \propto \epsilon^{-0.70} L^{-0.20}$, which clearly shows that ellipticity is in fact the primary parameter, having a stronger effect on the metallicity of dEs than the luminosity.

\section{Discussion of a possible explanation: Outflow of metal-rich gas along the minor axis}

The mass-metallicity relation of spheroidal galaxies, evident in Fig. 2, is most likely the result of early chemodynamical evolution. The central idea is gas loss by a galactic wind, which is believed to be particularly metal-enhanced (Vader 1986, 1987). Certainly, the mass (i.e. potential depth) of a galaxy must be a key parameter for the loss of gas (hence metals), and this explains the mass-metallicity relation. However, it has been pointed out by De Young \& Heckman (1994) that the shape (i.e. flattening) of a galaxy may play a crucial role as well. These authors show that galaxies of intermediate mass $\left(M \approx 10^{9} M_{\odot}\right)$ would not lose almost all of their ISM in a central starburst, but would preferentially undergo a blowout event, having gas flowing out in the direction of their minor axis. The important point is that the strength of such an outflow would critically depend on the intrinsic flattening of the ISM distribution, i.e. the galaxy. Our cluster dwarfs showing a metallicityflattening relation have masses precisely in the critical regime discussed by De Young \& Heckman (1994): their mass seems to be too low to be completely protected from gas loss (as in giant ellipticals), but also too high to experience a total blowaway (as in dwarf spheroidals). In the intermediate mass range 


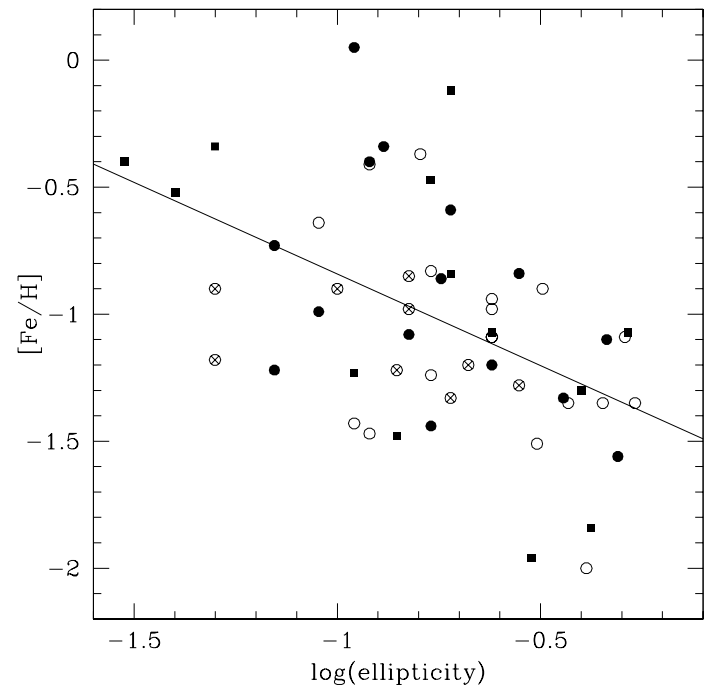

Fig. 5. $[\mathrm{Fe} / \mathrm{H}]$ versus log ellipticity for cluster $\mathrm{dEs}$. The line is a linear fit to the data (equation given in text). Symbols as in Fig. 2.

of bright cluster dEs the ellipticity could conceivably be the dominant parameter for gas loss, and hence metallicity.

Of course, reality must be more complicated. For one thing dwarf galaxies are believed to have large amounts of dark matter, so the gas escaping the stellar body should remain bound to the galaxy and be re-accreted on a rather short time scale (Mac Low \& Ferrara 1999; Ferrara \& Tolstoy 2000). In this context, it is interesting to note that we have found a possible difference between cluster dwarfs and (local) field dwarfs. While the evidence for dark matter in field dwarfs abounds, the situation with cluster galaxies in general is much less clear. $\mathrm{X}$-ray and lensing studies of clusters of galaxies suggest that the dark matter in clusters is not bound to individual galaxies, hence cluster dwarfs might be much less dark than field dwarfs. An additional difference is that cluster dwarfs are subject to ram-pressure stripping by the ICM, which might help to remove the gas flown out (e.g. Murakami \& Babul 1999).

\section{Conclusion}

We have presented evidence for a metallicity-flattening relation for dwarf elliptical galaxies based on colour and metallicity data available from the literature. At a given total magnitude, rounder $\mathrm{dEs}$ are more metal-rich. In the narrow magnitude range of bright cluster dwarfs $\left(-14 \gtrsim M_{B} \gtrsim-18\right)$, metallicity is more strongly correlated with ellipticity than luminosity (mass), i.e. ellipticity seems to be the primary parameter for the enrichment history of these galaxies. Possibly this holds only for cluster dwarfs; the effect is not significant for local dwarfs. A possible explanation is provided by the scenario of De Young \& Heckman (1994), where the outflow of gas, and hence the regulation of metallicity, depends on the intrinsic shape of a galaxy in the intermediate mass range around $10^{9} M_{\odot}$.

It would be highly desirable to strengthen the evidence with further observations, especially spectroscopically well determined metallicities for many cluster dwarfs. If confirmed, the effect is likely to be of importance for our understanding of the chemodynamical evolution of galaxies.

Acknowledgements. We are grateful to the Swiss National Science Foundation for financial support. We have made use of the LEDA database (http://leda.univ-lyon1.fr).

\section{References}

Bender, R., Burstein, D., \& Faber, S. M. 1993, ApJ, 411, 153

Binggeli, B., \& Popescu, C. C. 1995, A\&A, 298, 63

Brodie, J. P., \& Huchra, J. P. 1991, ApJ, 379, 157

Caldwell, N. 1983, AJ, 88, 804

Caldwell, N., \& Bothun, G. D. 1987, AJ, 94, 1116

Caldwell, N., Armandroff, T. E., Seitzer, P., \& Da Costa, G. S. 1992, AJ, 103, 840

De Young, D. S., \& Heckman, T. M. 1994, ApJ, 431, 598

Efstathiou, G., \& Fall, S. M. 1984, MNRAS, 206, 453

Faber, S. M. 1973, ApJ, 179, 731

Ferguson, H. C. 1994, in ESO/OHP Workshop on Dwarf Galaxies, ESO (Garching), ed. G. Meylan, \& P. Prugniel, 475

Ferrara, A., \& Tolstoy, E. 2000, MNRAS, 313, 291

Held, E. V., \& Mould, J. R. 1994, AJ, 107, 1307

Kobayashi, C., \& Arimoto, N. 1999, ApJ, 527, 573

Mac Low, M.-M., \& Ferrara, A. 1999, ApJ, 513, 142

Mateo, M. 1998, ARA\&A, 36, 435

Mould, J. R. 1984, PASP, 96, 773

Murakami, I., \& Babul, A. 1999, MNRAS, 309, 161

Peletier, R. F., Davies, R. L., Illingworth, G. D., Davis, L. E., \& Cawson, M. 1990, AJ, 100, 1091

Rakos, K., Schombert, J., Maitzen, H. M., Prugovecki, S., \& Odell, A. 2001, AJ, 121, 1974

Terlevich, R., Davies, R. L., Faber, S. M., \& Burstein, D. 1984, MNRAS, 196, 381

Vader, J. P. 1986, ApJ, 305, 669

Vader, J. P. 1987, ApJ, 317, 128

Visvanathan, N., \& Sandage, A. 1977, ApJ, 216, 214 